\title{
Summation-by-Parts Operators with Minimal Dispersion Error for Coarse Grid Flow Calculations
}

\author{
Viktor Linders, Marco Kupiainen and Jan Nordström \\ Journal Article
}

\section{Tweet}

N.B.: When citing this work, cite the original article.

Original Publication:

Viktor Linders, Marco Kupiainen and Jan Nordström, Summation-by-Parts Operators with Minimal Dispersion Error for Coarse Grid Flow Calculations, Journal of Computational Physics, 2017. 340, pp.160-176.

\section{http://dx.doi.org/10.1016/j.jcp.2017.03.039}

Copyright: Elsevier

http://www.elsevier.com/

Postprint available at: Linköping University Electronic Press

http://urn.kb.se/resolve?urn=urn:nbn:se:liu:diva-136474

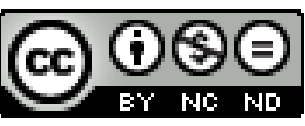




\title{
Summation-by-Parts Operators with Minimal Dispersion Error for Coarse Grid Flow Calculations
}

\author{
Viktor Linders $^{\mathrm{a}}$, Marco Kupiainen ${ }^{\mathrm{b}}$, Jan Nordström ${ }^{\mathrm{c}}$ \\ ${ }^{a}$ Department of Mathematics, Computational Mathematics, Linköping University, \\ SE-581 83 Linköping, Sweden (viktor.linders@liu.se). \\ ${ }^{b}$ Rossby Centre SMHI, SE-601 76 Norrköping, Sweden (marco.kupiainen@smhi.se). \\ ${ }^{c}$ Department of Mathematics, Computational Mathematics, Linköping University, \\ SE-581 83 Linköping, Sweden (jan.nordstrom@liu.se).
}

\begin{abstract}
We present a procedure for constructing Summation-by-Parts operators with minimal dispersion error both near and far from numerical interfaces. Examples of such operators are constructed and compared with a higher order non-optimised Summation-by-Parts operator. Experiments show that the optimised operators are superior for wave propagation and turbulent flows involving large wavenumbers, long solution times and large ranges of resolution scales.
\end{abstract}

Keywords: Summation-by-Parts; Dispersion relation; Finite differences; Wave propagation

\section{Introduction and motivation}

It is well known that maximising the formal order of accuracy with respect to the bandwidth of a finite difference stencil may lead to a suboptimal numerical scheme for problems involving high frequency waves over large time spans. In order to properly resolve such waves, a very small spatial increment is typically required. However, severe restrictions on the spatial increment naturally has a negative impact on the efficiency of the solver. Problems of this type are common in fields such as fluid dynamics, acoustics, meteorology, electromagnetism and seismology, and have been addressed accordingly, see e.g. $[1,2,3,4,5]$. For these problems, the errors in the numerical solutions are dominated by inaccurate approximations of the dispersion relations related to the governing partial differential equations. Wave properties encoded within 
the dispersion relation include phase velocity, group velocity, anisotropy and dissipation. It is therefore of interest to develop finite difference schemes that preserve the analytic dispersion relation of the governing equations for a wide range of spatial increments. In [6], a discussion and comparison is made of many stencils that attempt to do so. For more recent approaches, see e.g. $[7,8,9]$.

Discussions of numerical dispersion has in the literature largely been concerned with central finite difference stencils suitable for purely periodic problems. However, in realistic simulations numerical interfaces will generally be present, at which departure from centrality may be required, and questions of numerical stability must be addressed. In this paper we will present a procedure for designing new finite difference operators on Summation-by-Parts (SBP) form $[10,11,12,13,14]$ that approximate first derivatives both near and far from numerical interfaces. SBP operators combined with weakly imposed interface conditions lead to provably stable schemes, and may be constructed at high orders.

SBP operators based on dispersion relation preserving schemes [15] have previously been constructed in [16] and applied to the Euler and NavierStokes equations in $[17,18]$. However, in these works no particular procedure was applied in order to minimise the dispersion near interfaces. In this paper we will construct SBP operators based on the local stencils presented in [9], where the dispersion error of central difference stencils were minimised in the $L^{\infty}$-sense. We extend this work by analogously minimising the dispersion error of the non-central stencils arising near the interface. This minimisation problem is quasiconvex and may thus be solved fast and reliably using standard optimisation packages.

The SBP operators introduced in this paper are designed for problems where computational resources are a limiting factor. Their intended application is for problems where large wavenumbers are prevalent and grid refinement is not a viable option. As such, it is throughout this paper assumed that some prior knowledge of the spectral content of the solution is available. We will utilise this knowledge in the design of the SBP operators such that performance is improved for problems posed on grids that are coarse relative to the wavenumbers involved. The new operators have suboptimal order of accuracy relative to the bandwidth, leaving a set of free stencil parameters that are used to minimise the dispersion error. Each operator is therefore not intended to be used on a sequence of progressively finer grids. Rather, we introduce a procedure for obtaining a new SBP operator that is adjusted 
to the particular grid at hand.

This paper is structured as follows: We introduce relevant notation and mathematical concepts in Section 2. In Section 3 we discuss the definition, properties and structure Summation-by-Parts operators. We discuss the procedure of obtaining optimal SBP operators and show a few examples in Section 4. In Section 5 we illustrate the performance of the new SBP operators by solving relevant test problems. Finally, concluding remarks are made in Section 6.

\section{Preliminaries}

Before we proceed we introduce the notation and theoretical concepts that will be required.

\subsection{Notation}

Throughout this paper we will separate analytic quantities and approximated quantities, the latter of which we mark with an overbar. Thus, for example $\bar{\xi}$ would represent a numerical approximation of the analytic quantity $\xi$.

Matrices will in general be denoted by capitalised letters. Functions will be denoted by lower case letters, e.g. $u(x, t)$, unless otherwise specified. If a function is evaluated on a discrete grid we write it as a vector, e.g. $\mathbf{u}=$ $\left(u_{0}, \ldots, u_{N}\right)^{T}$. Here $u_{j}$ is the $j^{\text {th }}$ element of the $(N+1)$-dimensional vector $\mathbf{u}(t)$, given by the function value of $u(x, t)$ at the point $x=x_{j}, 0 \leq j \leq N$. In this paper we assume that all grids are equidistant and let $\Delta x=x_{j+1}-x_{j}$ be the spatial increment.

\subsection{Dispersion of local finite difference stencils}

Consider a local finite difference approximation at the point $x_{j}$ defined as a weighted sum of function evaluations at $L$ points to the left and $R$ points to the right of $x_{j}$;

$$
\left(\frac{\partial f}{\partial x}\right)_{j} \approx \frac{1}{\Delta x} \sum_{m=-L}^{R} c_{m} f_{j+m} .
$$

Here, $f_{j}$ is the $j$ th element of the vector $\mathbf{f}$, which is obtained by projecting the function $f(x)$ onto the grid, and $c_{m}$ denote the corresponding weights. 
Following the derivation in [15], we note that the above equation is a special case of

$$
\frac{\partial f(x)}{\partial x} \approx \frac{1}{\Delta x} \sum_{m=-L}^{R} c_{m} f(x+m \Delta x) .
$$

when $x=j \Delta x$. By the inverse Fourier transform we may write

$$
f(x)=\int_{-\infty}^{\infty} \hat{f}(\kappa) \exp (i \kappa x) \mathrm{d} \kappa,
$$

where $\hat{f}(\kappa)$ is the Fourier transform of $f$ and $\kappa$ is a wavenumber. Inserting this into (2), multiplying by $-i \Delta x$ and cancelling common terms gives

$$
\Delta x \kappa \approx-i \sum_{m=-L}^{R} c_{m} \exp (i m \Delta x \kappa) .
$$

Thus, the finite difference stencil provides an approximation of the analytic wavenumber in terms of a truncated Fourier-type series.

We introduce the notation $\xi=\kappa \Delta x$ as the normalised wavenumber. As the smallest resolvable wavelength is $\lambda_{\min }=2 \Delta x$, the largest resolvable wavenumber is $\kappa_{\max }=2 \pi / \lambda_{\min }=\pi / \Delta x$, implying that $|\xi| \leq \pi$. Typically we will consider some smaller range of wavenumbers, $\xi \in\left[0, \xi_{\text {max }}\right]$.

We define the dispersion error as

$$
E(\xi, \mathbf{c})=\xi-\bar{\xi}(\xi, \mathbf{c})=\xi-\left(-i \sum_{m=-L}^{R} c_{m} \exp (i m \xi)\right)
$$

where $\bar{\xi}(\xi, \mathbf{c})$ is the numerical approximation of the normalised wavenumber, henceforth referred to as the numerical wavenumber. The vector c contains the coefficients $c_{m}$.

We make a special note of the case when $L=R=n$, i.e. when the stencil is central. To distinguish this case from the non-central stencils we will refer to the vector of coefficients by the letter $\mathbf{a}$. In this case we have the relations $a_{m}=-a_{-m}, a_{0}=0$. Thus, by (4) the dispersion error for the central difference stencil is given by

$$
E(\xi, \mathbf{a})=\xi-2 \sum_{m=1}^{n} a_{m} \sin (m \xi) .
$$

In this case the numerical wavenumber is purely real. Hence, the stencil causes no growth or decay of the solution. 


\section{Summation-by-Parts operators}

Summation-by-Parts (SBP) operators were first derived in [10, 11] and further developed in [12]. Augmented with Simultaneous Approximation Terms (SAT) [19] that weakly enforce boundary conditions, the SBP-SAT technique allows for stable and high order discretisations of partial differential equations if well posed boundary conditions are available. For reviews of the SBP-SAT technique and its development, see [13, 20] and the references therein.

\subsection{Definition}

A Summation-by-Parts operator $D=P^{-1} Q$ may be defined by the properties

1. $\partial u /\left.\partial x\right|_{x=x_{j}} \approx \mathbf{e}_{j}^{T} D \mathbf{u}, \quad j=0, \ldots, N$,

2. $P=P^{T}>0$,

3. $Q+Q^{T}=\operatorname{diag}(-1,0, \ldots, 0,1)$,

where $P$ and $Q$ are matrices of dimension $(N+1) \times(N+1)$, and $\mathbf{u}$ is the projection of the function $u(x, t)$ onto the grid. Here, $\mathbf{e}_{j}$ is the $j$ th unit vector.

Remark 1. There are more general formulations than 1,2,3 above that extend the definition of Summation-by-Parts operators to non-cartesian grids, see [21, 22, 23].

\subsection{Stability}

To illustrate the use of the SBP-SAT technique we consider the periodic model problem

$$
\begin{array}{rlrl}
u_{t}+a u_{x} & =0, & x \in[0,1], & t \geq 0 \\
u(x, 0) & =f, & x \in[0,1] & \\
u(0, t) & =u(1, t), & t \geq 0,
\end{array}
$$

where we let $a>0$. Multiplying by $u$ and integrating in space gives the energy rate

$$
\frac{\mathrm{d}}{\mathrm{d} t}\|u\|^{2}=0
$$

which leads to a bounded solution, thus implying that the model problem is well posed. 
Consider now discretising in space. Rather than using a periodic operator we will introduce a numerical interface connecting the grid points at $x=0$ and $x=1$ by imposing periodic boundary conditions. A discretisation of (6) using an SBP operator yields

$$
\mathbf{u}_{t}+a P^{-1} Q \mathbf{u}=\sigma_{0} P^{-1}\left(u_{0}-u_{N}\right) \mathbf{e}_{0}+\sigma_{N} P^{-1}\left(u_{N}-u_{0}\right) \mathbf{e}_{N},
$$

where the right-hand side consists of SAT terms that weakly impose the periodic interface condition at the first and last grid points in the domain. Here, $\mathbf{e}_{0}$ and $\mathbf{e}_{N}$ are the first and last columns of the identity matrix with dimension $N+1$. Multiplying by $\mathbf{u}^{T} P$ and adding the transpose of the resulting equality yields

$$
\frac{\mathrm{d}}{\mathrm{d} t}\|\mathbf{u}\|_{P}^{2}=u_{0}^{2}\left(2 \sigma_{0}+a\right)+u_{N}^{2}\left(2 \sigma_{N}-a\right)-2 u_{0} u_{N}\left(\sigma_{0}+\sigma_{N}\right),
$$

where we have used point 2 in the definition of the SBP operator to define the norm $\|\mathbf{u}\|_{P}^{2}$ as $\mathbf{u}^{T} P \mathbf{u}$. Additionally, we have used point 3 in the definition in order to extract the boundary terms from $Q$. In order to bound the right hand side we let the penalty parameters take the form [24]

$$
\sigma_{0}=-\frac{a+\theta}{2}, \quad \sigma_{N}=\frac{a-\theta}{2},
$$

for some constant $\theta$. Inserting this into (9) and simplifying gives the final discrete energy rate

$$
\frac{\mathrm{d}}{\mathrm{d} t}\|\mathbf{u}\|_{P}^{2}=-\theta\left(u_{0}-u_{N}\right)^{2},
$$

which is bounded if $\theta \geq 0$. Thus the discretisation (8) is a stable approximation of the model problem (6). Note that the discrete energy rate (10) mimics the continuous one (7) exactly if $\theta=0$, and adds a small dissipative term otherwise.

\subsection{Structure and accuracy}

As an example of the typical structure of $Q$ we consider

$$
Q=\left(\begin{array}{ccccccc}
-1 / 2 & q_{12} & q_{13} & q_{14} & & & \\
-q_{12} & 0 & q_{23} & q_{24} & & & \\
-q_{13} & -q_{23} & 0 & q_{34} & a_{2} & & \\
-q_{14} & -q_{24} & -q_{34} & 0 & a_{1} & a_{2} & \\
& & -a_{2} & -a_{1} & 0 & a_{1} & a_{2} \\
& & & & & \ddots &
\end{array}\right)
$$


where $\mathbf{a}=\left(a_{1}, a_{2}\right)$ is the vector of coefficients of a (given) central difference stencil, and $q_{i j}$ are stencil coefficients to be determined. Thus, $Q$ consists of an interior set of central difference stencils augmented with blocks of size $r \times r$ to account for the discretisation near domain interfaces.

For stability reasons $[25,26]$ we will in this paper limit our analysis to the case when $P$ is a diagonal matrix of the form

$$
P=\Delta x \operatorname{diag}\left(p_{1}, \ldots, p_{r}, 1, \ldots, 1, p_{r}, \ldots, p_{1}\right),
$$

where $p_{j}, j=1, \ldots, r$ are positive real numbers.

In order for the matrices $P$ and $Q$ to be compatible they must satisfy certain linear accuracy conditions, first described in [10]. Let us impose that $D=P^{-1} Q$ should exactly differentiate polynomials up to degree $p$. Then, for the monomial $\mathbf{x}^{j}$ (where the exponent is to be taken elementwise and $\mathbf{x}^{-1}=\mathbf{0}$, where $\mathbf{0}$ is the all zeros vector), we have

$$
Q \mathbf{x}^{j}=j P \mathbf{x}^{j-1}, \quad j=0, \ldots, p .
$$

Multiplying (11) from the left by $\left(\mathbf{x}^{i}\right)^{T}$ and adding the resulting equality with swapped indices gives the identity

$$
\begin{aligned}
\left(\mathbf{x}^{i}\right)^{T} Q \mathbf{x}^{j}+\left(\mathbf{x}^{j}\right)^{T} Q \mathbf{x}^{i} & =j\left(\mathbf{x}^{i}\right)^{T} P \mathbf{x}^{j-1}+i\left(\mathbf{x}^{j}\right)^{T} P \mathbf{x}^{i-1} \\
& =(i+j) \mathbf{1}^{T} P \mathbf{x}^{i+j-1},
\end{aligned}
$$

where $\mathbf{1}$ is the all ones vector and we have used the diagonal structure of $P$ in the final equality. Let $s=i+j$. Simplifying using property 3 in the definition of the SBP operator gives

$$
s \mathbf{1}^{T} P \mathbf{x}^{s-1}=x_{N}^{s}-x_{0}^{s}, \quad s=1, \ldots 2 p .
$$

Note that (12) defines a set of $2 p$ linear conditions on $P$ (the case $s=0$ is trivially satisfied). In applications we will typically rearrange these conditions such that they take the simpler form

$$
A_{0} \mathbf{p}=\mathbf{b}_{0},
$$

where the vector $\mathbf{p}$ contains the diagonal elements in $P$.

Consider now $Q$. Assuming that $P$ has been determined by solving (13), the equality (11) defines a set of linear accuracy conditions on the elements in $Q$. With a suitable rearrangement this may be written in the form,

$$
A_{1} \mathbf{q}=\mathbf{b}_{1}
$$

where the vector $\mathbf{q}$ contains the elements of $Q$. 
Remark 2. If the interior stencil a is predefined, the conditions (13) and (14) can generally be written down only for the $r$ rows near the interface. For details of how to obtain such restricted conditions, see [10].

If the interior stencil a of $D=P^{-1} Q$ has formal order of accuracy $\mathcal{O}\left(\Delta x^{2 p}\right)$, then the interface block can only reach accuracy of order $\mathcal{O}\left(\Delta x^{p}\right)$ [10]. It can be shown that this leads to solutions with global order $\mathcal{O}\left(\Delta x^{p+1}\right)$ if the approximation is pointwise stable and the problem is first order hyperbolic [27].

In summary, given a desired order of accuracy $p$, an interior stencil $\mathbf{a}$, and a given dimension of the interface block $r$, a Summation-by-Parts operator $D$ may be constructed by satisfying the accuracy conditions on $P$ and $Q$ simultaneously while ensuring that $P$ is positive definite. Certainly for many (in fact most) feasible parameter choices there will be some degrees of freedom left after all conditions are satisfied. In the next section we will utilise this freedom in order to minimise the dispersion errors of each row at the interface block of $D$.

\section{SBP operators with minimal dispersion errors}

We now turn to the problem of obtaining SBP operators with minimal dispersion errors. It should be noted that defining the objective function for the minimisation can be done in many different ways. Here, we attempt to define this function in a way analogous to the optimisation that was performed in [9] for central difference stencils.

\subsection{Optimisation procedure}

We begin by assuming that the partial differential equation that is to be approximated is either periodic or has initial data given on the whole real line, such that it is amenable to Fourier analysis. Then the solution may be expressed in terms of a series of Fourier modes, each of the form $f(x, t)=$ $\exp (i(\kappa x-\omega t))$, where $\kappa$ and $\omega$ respectively denote the wavenumber and frequency of the mode.

Remark 3. Note that interfaces present in the numerical scheme are absent in the continuous setting, hence the above considerations are valid.

We now consider a semi-discrete setting where time is left continuous while space is discretised on a suitable grid, and where one or several numerical interfaces are introduced. In the presence of an interface, Fourier 
transforming the semi-discrete operator may not be a valid approach. Instead, we will utilise $f(x, t)$ as a target function for a global minimisation problem as follows:

Let $\mathbf{f}(t)$ denote the projection of $f$ onto the spatial grid. We require $D \mathbf{f}=P^{-1} Q \mathbf{f}$ to be a good approximation of the derivative operation applied to $f$ and consider the residual vector

$$
\mathbf{r}(\xi)=i \kappa \mathbf{f}-D \mathbf{f}=\frac{i}{\Delta x}(\xi \mathbb{I}-\operatorname{diag}(\boldsymbol{\xi})) \mathbf{f} .
$$

Here, $\mathbb{I}$ is the identity matrix. The elements of the vector $\boldsymbol{\xi}$ are the numerical wavenumbers of each row in $D$ as defined in (3). Our task is to minimise $\mathbf{r}(\xi)$ with respect to some suitable norm.

We simplify the task by assuming that the interior stencil is given such that we only need to consider the interface blocks present in $D$. Here we will use the optimised central difference stencils introduced in [9], for which the dispersion error is minimised in the $L^{\infty}$ sense. We further assume that all interfaces are treated in the same way such that we only need to consider one block. As before, we let $r$ be the number of rows in the interface block, and let $\mathbf{q}$ and $\mathbf{p}$ be vectors containing the interface coefficients of $Q$ and $P$ respectively. The problem at hand may now be formulated as

$$
\begin{aligned}
& \text { Find } \quad \arg \min _{\mathbf{q}, \mathbf{p}} \max _{j \in \mathcal{J}_{r}}\left\|r_{j}(\xi)\right\|_{\infty} \\
& \text { subject to } \quad A_{0} \mathbf{p}=\mathbf{b}_{\mathbf{0}} \\
& \text { and } \quad A_{1} \mathbf{q}=\mathbf{b}_{\mathbf{1}}
\end{aligned}
$$

where $\mathcal{J}_{r}$ is the set of row indices corresponding to the rows of the interface block under consideration. Here, $r_{j}(\xi)$ is the dispersion error of the form (4) associated with row $j$ and corresponds to the $j$ th element of the residual vector $\mathbf{r}(\xi)$ defined in (15). The notation $\|\cdot\|_{\infty}$ refers to the $L^{\infty}$ norm, chosen to fit the interior stencils developed in [9]. Finally, $A_{0}, A_{1}, \mathbf{b}_{\mathbf{0}}$ and $\mathbf{b}_{\mathbf{1}}$ correspond to the conditions (13) and (14) ensuring accuracy $\mathcal{O}\left(\Delta x^{p+1}\right)$ of the resulting scheme.

Remark 4. In what follows we will restrict our attention to the real part of the dispersion errors as this determines the speed of the propagating solution. The imaginary part locally affects the amplitude of the solution. However, as seen from the discrete energy rate (10), the change in energy of the solution is unaffected by the specific stencil parameters. Thus, even if some small 
redistribution of energy among modes can occur, we will henceforth ignore the imaginary part of the dispersion errors.

With a slight abuse of notation, we let the real part of the dispersion error at the $j$ th row of the boundary block of $D$ be given by

$$
E_{j}(\xi, \mathbf{q}, \mathbf{p})=\xi-\bar{\xi}_{j}(\xi, \mathbf{q}, \mathbf{p}),
$$

where $\bar{\xi}_{j}(\xi, \mathbf{q}, \mathbf{p})$ is the real part of the numerical wavenumber of the stencil at row $j$. It is obvious from (15) that the solution of (16) is the same as that of the following problem:

$$
\begin{array}{lrl} 
& \text { Find } & \arg \min _{\mathbf{q}, \mathbf{p}} \max _{j \in \mathcal{J}_{r}}\left\|E_{j}(\xi, \mathbf{q}, \mathbf{p})\right\|_{\infty} \\
\text { subject to } & A_{0} \mathbf{p}=\mathbf{b}_{\mathbf{0}} \\
\text { and } & A_{1} \mathbf{q}=\mathbf{b}_{\mathbf{1}}
\end{array}
$$

where the quantities involved are defined in the same way as in (16). In words, we track all $r$ dispersion errors near the interface simultaneously and look for the choice of $\mathbf{q}$ and $\mathbf{p}$ that makes the largest among them as small as possible.

Remark 5. The SBP operators are obtained by requiring that they should accurately approximate the derivatives of certain Fourier modes. In contrast to the derivation of the dispersion error (4), this requires no Fourier analysis in the semi-discrete setting. However, as (15), (16) and (17) shows, this approach is equivalent to treating each row of $D$ as a separate finite difference operator and subsequently applying Fourier analysis.

In order to solve (17) with reasonable efficacy we let $\mathbf{x}^{T}=\left(\mathbf{q}^{T}, \mathbf{p}^{T}\right)$ and define the objective function

$$
\phi(\mathbf{x})=\max _{j \in \mathcal{J}_{r}}\left\|E_{j}(\xi, \mathbf{x})\right\|_{\infty}=\max _{j \in \mathcal{J}_{r}} \sup _{\xi \in\left[0, \xi_{\text {max }}\right]}\left|E_{j}(\xi, \mathbf{x})\right|,
$$

where $\xi_{\max } \leq \pi$ is the upper limit of the wavenumber domain of interest. Problem (17) can now be written

$$
\begin{array}{lr}
\text { Find } & \arg \min _{\mathbf{x}} \phi(\mathbf{x}) \\
\text { subject to } & A \mathbf{x}=\mathbf{b}
\end{array}
$$


where $A$ and $\mathbf{b}$ are suitable concatenations of $A_{0}, A_{1}, \mathbf{b}_{\mathbf{0}}$ and $\mathbf{b}_{\mathbf{1}}$.

Recall from (4) that $E_{j}$ takes the form

$$
E_{j}=\xi-\left(-i \sum_{m=-L}^{R} c_{m} \exp (i m \xi)\right)
$$

Here, $c_{m}$ are the stencil coefficients of $D=P^{-1} Q$ at row $j$ of the interface block. Thus, $c_{m}=q_{j m} / p_{j}$ and hence, $E_{j}$ is a linear function of the elements in $Q$ and an inversely linear function of the strictly positive elements in $P$. Such a function is quasiconvex (see [28] for a definition and details). Quasiconvexity is preserved both under the supremum and the maximum operations, and thus $\phi(\mathbf{x})$ is a quasiconvex function of $\mathbf{x}$.

There are many algorithms for solving the quasiconvex problem (18). For a review, see e.g. [29]. Here we have used an approach based on the repeated solution of associated convex programs, outlined in [28], which merely requires a convex program solver. To this end we have used the CVX package $[30,31]$, which allows us to obtain optimised SBP operators, typically in a matter of seconds. ${ }^{1}$

\subsection{Examples of SBP operators}

We have seen that SBP operators may be defined through the triplet $(p, r, \mathbf{a})$ where, as before, $p$ is the order of accuracy of the resulting operator, $r$ is the dimension of the matrix block near the interface, and a contains the coefficients of the repeated interior stencil, which is of order $2 p$. Recall that our goal is to obtain operators with improved performance for problems involving large wavenumbers relative to $\Delta x$, and where grid refinement is not a viable option. The best choice of $(p, r, \mathbf{a})$ is in general highly dependent on the problem at hand and the computational resources available.

In Section 5 we will compare the performance of new optimised SBP operators with a higher order classical (in the sense of non-optimised) operator available in the literature [32]. This operator will be denoted $\operatorname{SBP}(8,4)$ as it is fourth order accurate (i.e. $p=4$ ) near the interface and eighth order in the interior. This stencil has an interface block of dimension $8 \times 8$ (i.e.

\footnotetext{
${ }^{1} \mathrm{~A}$ Matlab code generating the interior stencils, a, and the boundary coefficients, $\mathbf{p}$ and $\mathbf{q}$, is available. Please contact the first author of this paper for details.
} 


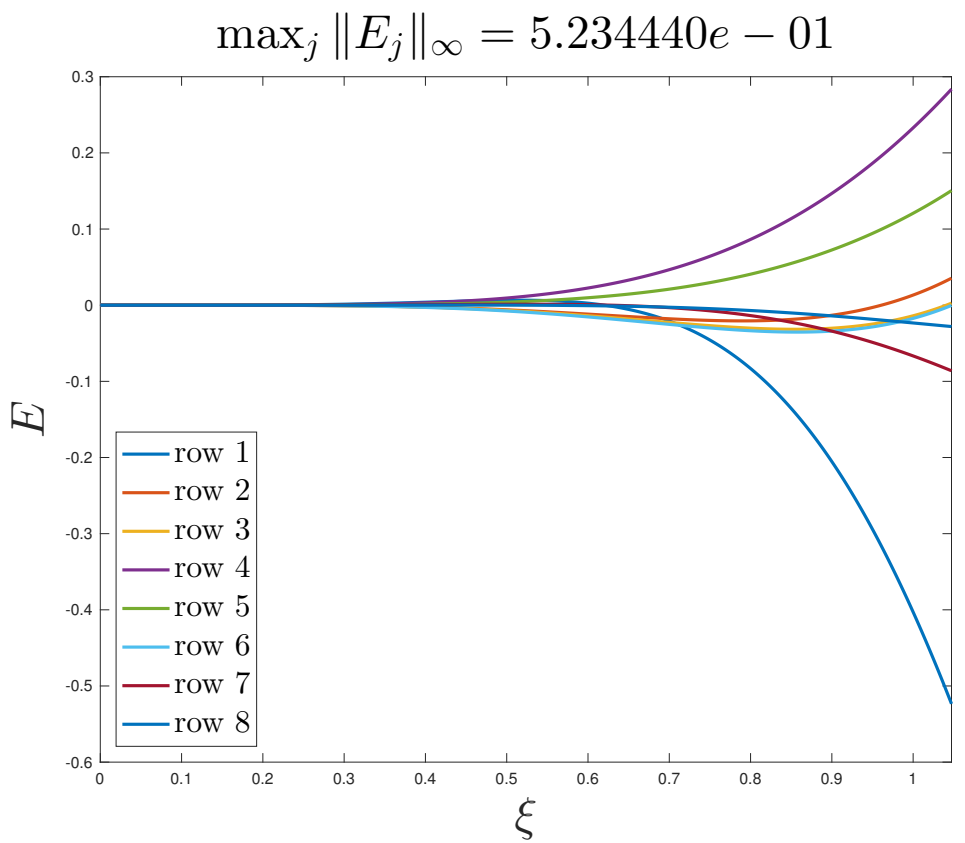

(a) $\operatorname{SBP}(8,4)$

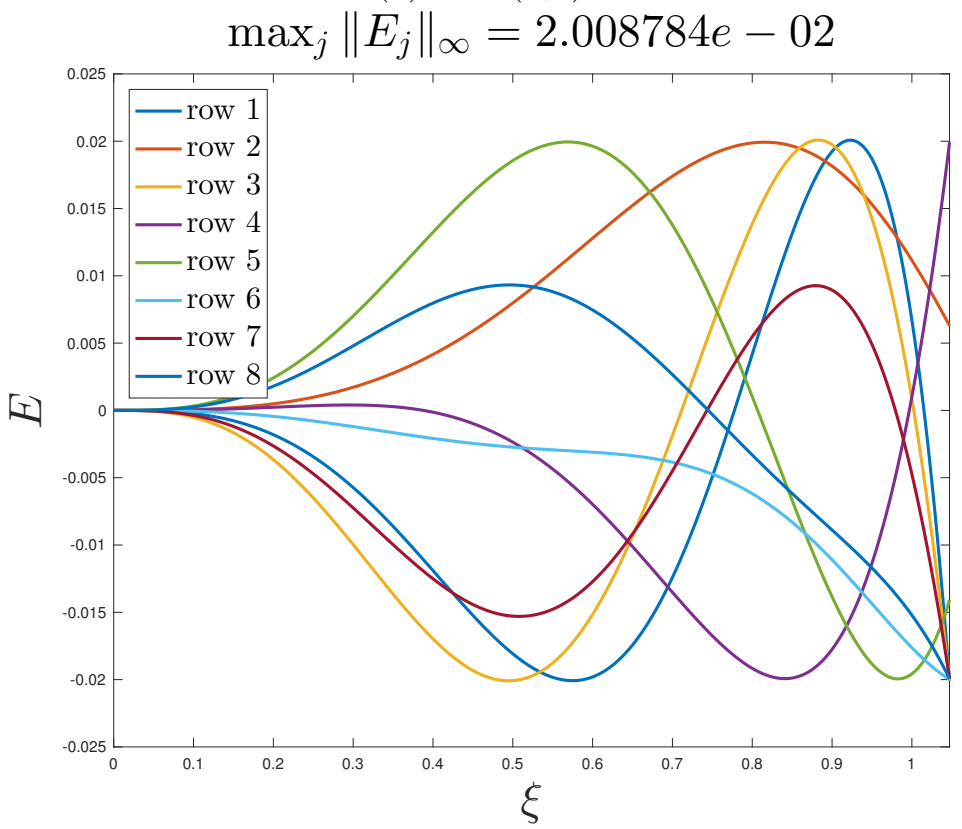

(b) $\operatorname{SBP}(4,2,2,8, \pi / 3)$

Figure 1: Dispersion errors of SBP operators. 


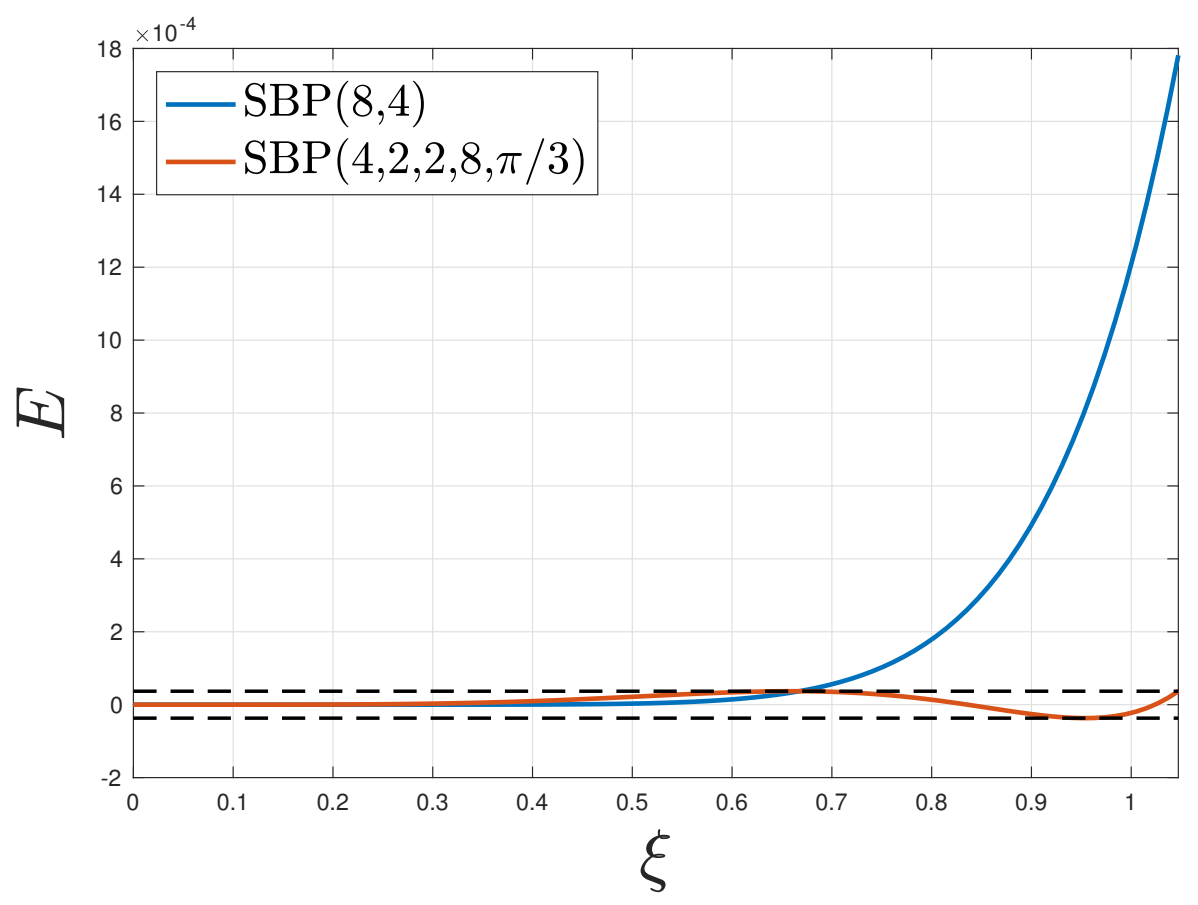

Figure 2: Dispersion errors of interior stencils.

$r=8)$. It should be noted that there are three free parameters in the interface block of $\operatorname{SBP}(8,4)$. These are chosen in such a way that the spectral radius is minimised.

New optimised operators will be denoted $\operatorname{SBP}\left(2 p, p, n, r, \xi_{\text {max }}\right)$ where $p$, $r$ and $\xi_{\max }$ are defined as previously and $n$ denotes the number of free parameters introduced in the interior stencil. The cost of using a given SBP operator depends on its sparsity, which in turn depends on $r$ and the total bandwidth of the interior stencil, given by $2(p+n)+1$. For $\operatorname{SBP}(8,4)$ this amounts to $9(p=4$ and $n=0)$. For fair comparisons, we will here only consider optimised SBP operators of bandwidth 9 (i.e. $p+n=4$ ). Likewise, we will constrain ourselves to using operators with $r=8$. Thus, the computational complexity of using each SBP operator is the same throughout this paper.

Examples of operators are shown in Fig. 1, where the dispersion errors of the first eight rows of $\operatorname{SBP}(8,4)$ and $\operatorname{SBP}(4,2,2,8, \pi / 3)$ are plotted. Note that the largest error of $\operatorname{SBP}(4,2,2,8, \pi / 3)$ is reduced by a factor of 25 compared to 
$\operatorname{SBP}(8,4)$. In Fig. 2 the dispersion errors of the interior stencils are shown. Here, the $L^{\infty}$ norm dispersion error of $\operatorname{SBP}(8,9)$ is about $1.8 \times 10^{-3}$ whereas that of $\operatorname{SBP}(4,2,2,8, \pi / 3)$ is $3.7 \times 10^{-5}$; an improvement by a factor close to 50. Fig. 2 reveals that a small sacrifice has been made for $\operatorname{SBP}(4,2,2,8, \pi / 3)$ at low wavenumbers in order to achieve the error reduction at the higher ones. In fact, $\operatorname{SBP}(8,4)$ may be considered the optimal operator in the limit $\xi_{\max } \rightarrow 0$. For details on this, see [9].

\subsection{Limitations}

As previously stated, the optimal choice of the triplet $(p, r, \mathbf{a})$ depends on the computational resources and the target problem to be solved. Depending on the situation, different SBP operators may have various advantages or disadvantages that govern the choice of the final scheme.

In Fig. 3 the spectral radius of a large sample of different SBP operators are shown, including second (black), fourth (red) and sixth (magenta) order operators optimised with $\xi_{\max } \in\{\pi / 2, \pi / 3, \pi / 4, \pi / 5, \pi / 6\}$. $\operatorname{SBP}(8,4)$ is shown in blue. Here we have multiplied the operator by $\Delta x$ to aid the visualisation. Clearly, most operators have very similar spectral radii, the obvious exception being $\operatorname{SBP}(4,2,2,8, \pi / 3)$, which has a spectral radius about four times that of $\operatorname{SBP}(8,4)$. Thus, for a situation where computational speed is an issue, $\operatorname{SBP}(4,2,2,8, \pi / 3)$ may not be the right choice since it would require a comparably small temporal step size to satisfy the CFL condition. This drawback can certainly be remedied, e.g. by introducing a constraint on the size of the elements in the matrices $P$ and $Q$ in the optimisation procedure. We have refrained from doing so here for the purpose of illustration.

Additional specialisations may be considered such as departing from uniform grids in order to reduce the dispersion error further. This was done for classical SBP operators in [33] in an attempt to minimise the truncation error. This approach introduces extra degrees of freedom in the optimisation problem; however, in a non-linear way. The optimisation procedure would have to be altered to incorporate this non-linearity, hence we will not consider such operators in this paper.

\section{Numerical examples}

We proceed by illustrating the performance of the optimised SBP operators compared to the classical one through three numerical examples. The first is a narrow Gaussian pulse solution to the one-dimensional advection 


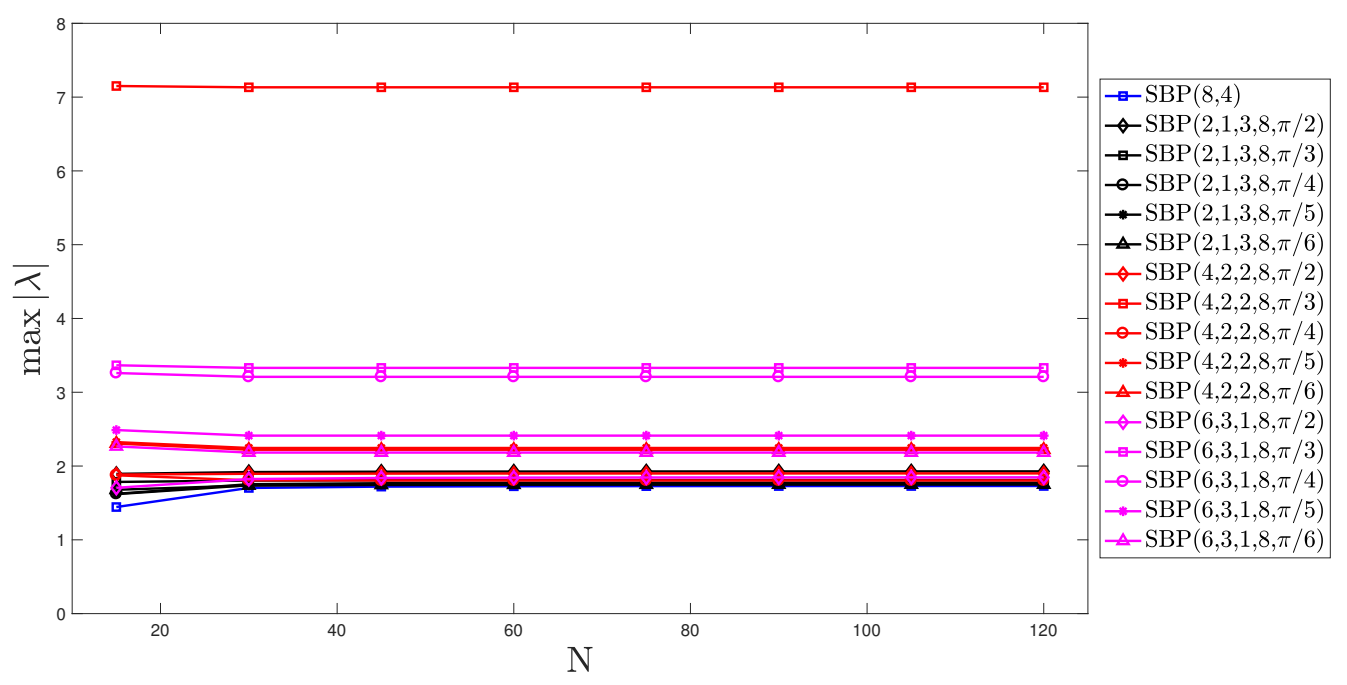

Figure 3: Spectral radius of various SBP operators.

equation. This simple illustration exemplifies the use of optimised operators for solutions with very high wavenumber content. The second example is the propagation of a vortex governed by the Euler equations, which is a common example of a non-linear problem exhibiting unphysical dispersive phenomena if not properly resolved [34, 9]. This example illustrates the impact of the dispersion error on long-time simulations. The third example is a calculation of the Taylor-Green vortex using the Navier-Stokes equations, which demonstrates that optimised operators may be used with less artificial dissipation than classical ones, which is advantageous in simulations involving a large range of resolution scales.

\subsection{Gaussian pulse}

Consider the periodic advection equation

$$
u_{t}+u_{x}=0, \quad-3.5 \leq x \leq 3.5, \quad t \geq 0
$$

with exact solution

$$
u(x, t)=2 \exp \left(-3200(x-t+3)^{2}\right) .
$$

This Gaussian pulse is narrow and thus its Fourier transform is wide resulting in a significant contribution from a broad range of wavenumbers. It 
should be noted that the Fourier transform is also a Gaussian and thus small wavenumbers somewhat dominate larger ones.

We introduce interfaces at $x= \pm 1$, thus dividing space into three regions, $\Omega_{i}, i \in\{1,2,3\}$, as illustrated in Fig. 4. We impose the condition that the solution should not vary across each interface. This problem is identical to (6) apart from the number and location of the interfaces. Thus, we can obtain a stable and energy conservative discretisation using the SBP framework with SAT penalty terms parametrised with $\theta=0$; see (10).

In $\Omega_{1}$ and $\Omega_{3}$ we use a grid with $\Delta x=1 / 100$ and utilise operators optimised with $\xi_{\max }=\pi / 2$. In $\Omega_{2}$ we use a slightly finer grid with $\Delta x=$ $1 / 125$ and accordingly choose operators with a smaller optimisation range, $\xi_{\max }=\pi / 3$. We integrate in time using the classical fourth order RungeKutta scheme with time step $\Delta t=10^{-3}$ so that the contribution to the error from the temporal discretisation is insignificant. The numerical solutions are shown in Figure 5. Here, we have solved the problem using second (magenta), fourth (orange) and sixth (red) order operators, optimised for the wavenumbers just indicated. The problem has also been solved using the classical $\operatorname{SBP}(8,4)$ in all three domains. For clarity, the pulses have been separated vertically.

Fig. 5 reveals two discernible errors. The first is the train of small pulses appearing behind and ahead of the main peak, resulting from high wavenumber modes travelling with an incorrect speed. The extent of this effect is seen by the decay of amplitude of the main peak, which is pronounced for $\operatorname{SBP}(8,4)$ and the sixth order operators, but noticeably less in the fourth and second order cases. In [9] it was shown that classical central difference operators (such as the interior stencil of $\operatorname{SBP}(8,4)$ ) underestimate the wavespeed of Fourier modes of any wavenumber greater than zero. There are no pulses ahead of the main peak for the $\operatorname{SBP}(8,4)$ solution suggesting that this effect

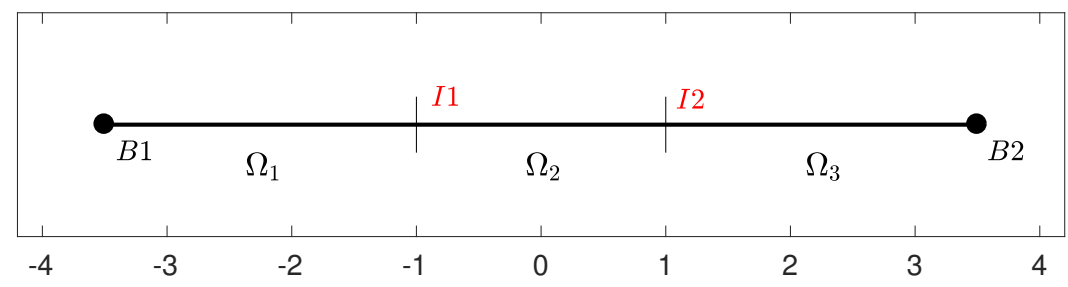

Figure 4: Geometry of the advection problem. 


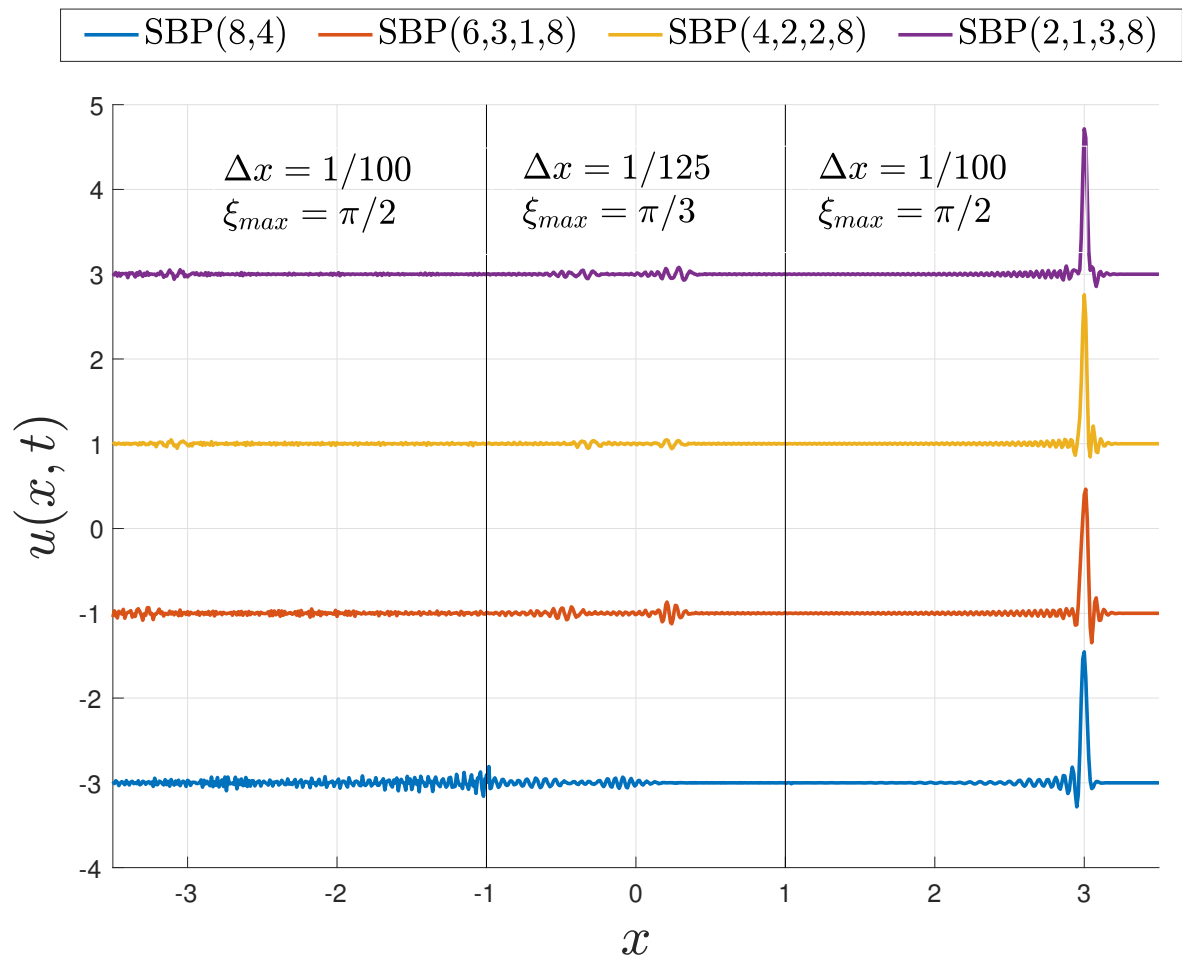

Figure 5: Numerical solutions at time $t=6$.

is primarily caused by the interior stencil of the SBP operators.

The second error takes the form of oscillatory noise left behind the main peak in $\Omega_{1}$ and $\Omega_{2}$. This is caused by reflections of unresolved, high wavenumber content in the solutions as they pass through the interfaces. Clearly this effect is significantly more pronounced for the $\operatorname{SBP}(8,4)$ operator than the optimised ones. Though much of the noise will eventually leave the domain through the left boundary, spurious oscillations are still clearly visible long after the main peak has passed.

The fact that the lower order operators appear to perform better in this experiment should come as no surprise as they have more degrees of freedom to be used in the optimisation. On a different grid, care must be taken to use operators that are optimised for the wavenumbers involved. Fig. 6 and Fig. 7 show convergence plots for $\operatorname{SBP}(8,4)$ and the optimised operators in two different situations. The same operator combinations that were considered 


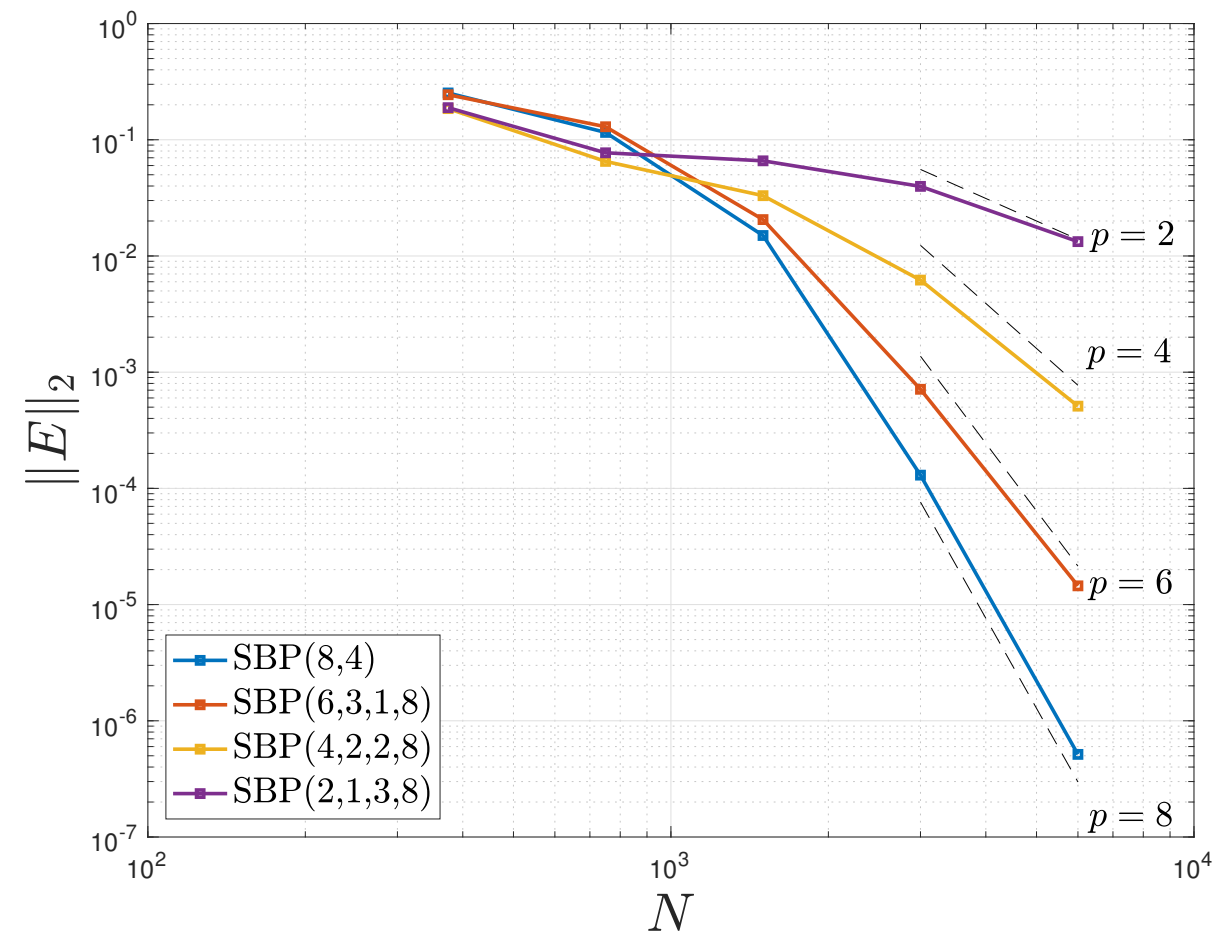

Figure 6: Error convergence of SBP operators. The optimisation only accounts for the coarsest grids.

in Fig. 5 are used on a sequence of progressively finer grids to obtain Fig. 6 . Here, Fig. 5 correspond to the second data point. Clearly $\operatorname{SBP}(8,4)$ is the better performer on the finer grids. This is expected, partly due to the faster convergence rate and partly since the other operators are not appropriately optimised for the small wavenumbers that dominate the solution on the finer grids.

In Fig. 7 we have used a sequence of operators optimised using smaller $\xi_{\max }$ on finer grids. By doing so, the operators are adapted to the smaller wavenumbers that become increasingly dominant as the grid is refined (recall that $\xi$ scales as $\Delta x$ ). In this case, the optimised operators are the better performers on a larger range of grids than previously, though on very fine grids, high order is still the better choice. Note that the errors of the optimised operators have been reduced by between one and two orders of magnitude 


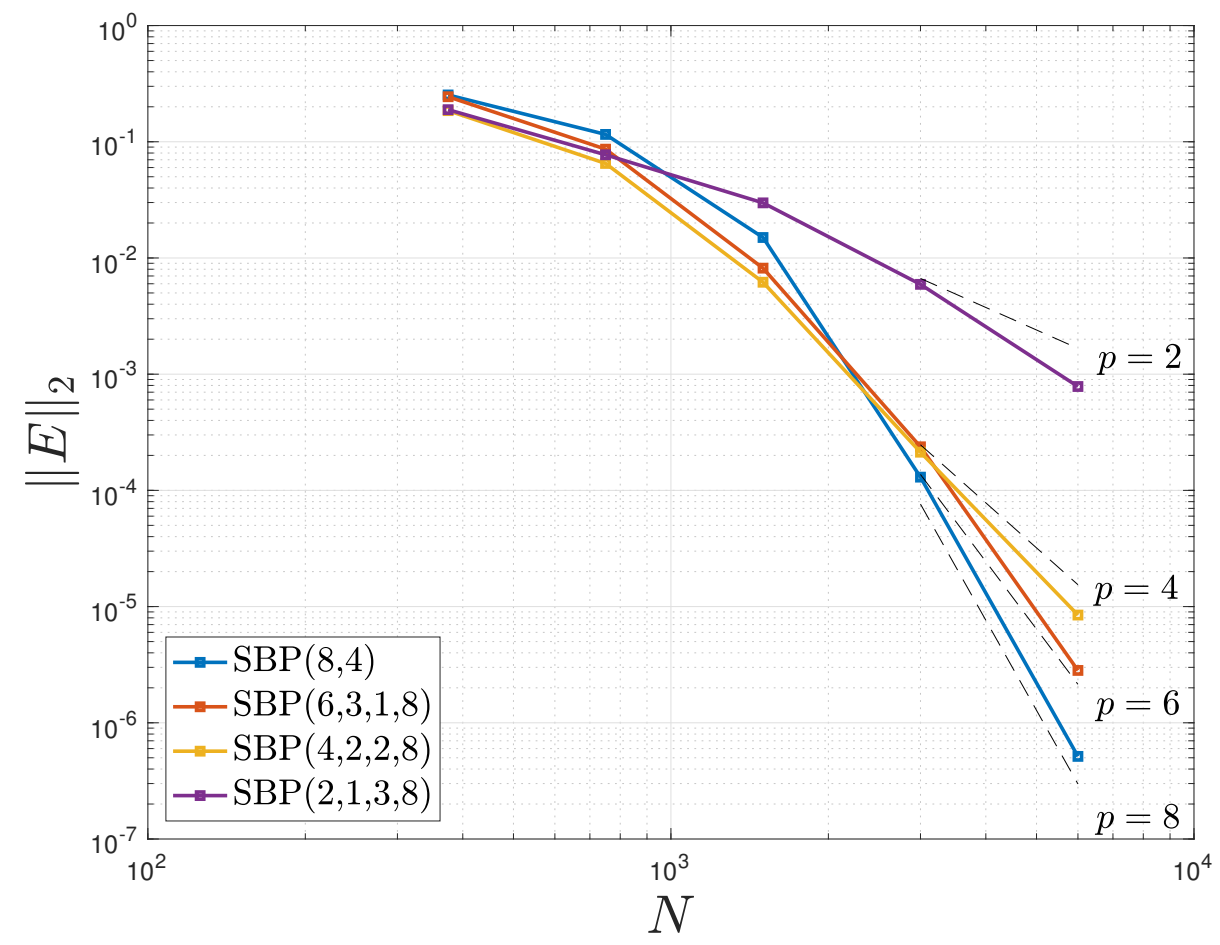

Figure 7: Error convergence of SBP operators. The optimisations is adapted to the finer grids.

on the finest grids.

It is interesting to note that the asymptotic convergence rate of all operators are closer to that of the interior stencil than of the interface stencil. This suggests that, for this particular problem, the impact of the interfaces become negligible in the asymptotic regime.

\subsection{The Euler vortex}

The compressible Euler equations in two dimensions, written in conservative form, are given by

$$
U_{t}+F_{x}+G_{y}=0
$$


where

$$
U=\left(\begin{array}{c}
\rho \\
\rho u \\
\rho v \\
e
\end{array}\right), \quad F=\left(\begin{array}{c}
\rho u \\
\rho u^{2}+p \\
\rho u v \\
u(e+p)
\end{array}\right), \quad G=\left(\begin{array}{c}
\rho v \\
\rho u v \\
\rho v^{2}+p \\
v(e+p)
\end{array}\right) .
$$

Here, $\rho$ is the density; $u$ and $v$ are the velocity components in the $x$ - and $y$-directions respectively; $e$ is the the total energy density; $p$ is the pressure, related to the conserved variables through the equation of state,

$$
p=(\gamma-1)\left(e-\frac{1}{2} \rho\left(u^{2}+v^{2}\right)\right)
$$

where $\gamma=1.4$ is the ratio of specific heats.

In [34] a vortex model that solves (19) was introduced. The model assumes a uniform background flow along the $x$-axis with pressure $P_{\infty}$, temperature $T_{\infty}$ and a Mach number $M_{\infty}$. A vortical movement, characterised by the radius $R$ and the vortex strength $\beta$, centered around the coordinates $\left(x_{0}, y_{0}\right)$, is superimposed. The local velocity components and the temperature assume the form

$$
\begin{aligned}
u & =M_{\infty}\left(1-\beta \frac{y-y_{0}}{R} \exp \left(-r^{2} / 2\right)\right) \\
v & =M_{\infty} \beta \frac{x-x_{0}}{R} \exp \left(-r^{2} / 2\right) \\
T & =T_{\infty}-\frac{\gamma-1}{2} M_{\infty}^{2} \beta^{2} \exp \left(-r^{2}\right),
\end{aligned}
$$

where $r^{2}=R^{-2}\left(\left(x-x_{0}\right)^{2}+\left(y-y_{0}\right)^{2}\right)$.

The Euler vortex is largely dominated by small wavenumbers, though with an increasing contribution from higher ones as the vortex strength, $\beta$, is increased, as well as when coarse grids are used. In the following experiment we use a moderately strong vortex with $\beta=2.5$ while setting $M_{\infty}=R=0.5$. We use a square domain with $x \in[0,10], y \in[-5,5]$ and periodic boundaries.

The problem is discretised on three different grids, respectively made up of 64,128 and 256 grid points in each direction. We solve the problem using $\operatorname{SBP}(8,4)$ and the optimised operators $\operatorname{SBP}\left(4,2,2,8, \xi_{\max }\right)$, where $\xi_{\text {max }} \in$ $\{\pi / 4, \pi / 5, \pi / 6\}$. The periodic boundary conditions are enforced using the SBP-SAT framework by introducing numerical interfaces in the same fashion as for the advection problem. For the temporal discretisation we use an 


\begin{tabular}{|c|c|c|c|}
\hline \multirow{4}{*}{$\operatorname{SBP}(8,4)$} & Grid & $L^{2}$ error & $p$ \\
\hline & $64 \times 64$ & $2.94 \times 10^{-3}$ & \multirow{3}{*}{$\begin{array}{l}3.40 \\
4.40\end{array}$} \\
\hline & $128 \times 128$ & $2.79 \times 10^{-4}$ & \\
\hline & $256 \times 256$ & $1.32 \times 10^{-5}$ & \\
\hline \multirow{4}{*}{$\operatorname{SBP}(4,2,2,8, \pi / 4)$} & Grid & $L^{2}$ error & $p$ \\
\hline & $64 \times 64$ & $3.34 \times 10^{-3}$ & \multirow{3}{*}{$\begin{array}{l}3.02 \\
3.46\end{array}$} \\
\hline & $128 \times 128$ & $4.12 \times 10^{-4}$ & \\
\hline & $256 \times 256$ & $3.75 \times 10^{-5}$ & \\
\hline \multirow{4}{*}{$\operatorname{SBP}(4,2,2,8, \pi / 5)$} & Grid & $=L^{2}$ error & $p$ \\
\hline & $64 \times 64$ & $4.60 \times 10^{-3}$ & \multirow{3}{*}{$\begin{array}{l}3.13 \\
3.37\end{array}$} \\
\hline & $128 \times 128$ & $5.25 \times 10^{-4}$ & \\
\hline & $256 \times 256$ & $5.07 \times 10^{-5}$ & \\
\hline \multirow{4}{*}{$\operatorname{SBP}(4,2,2,8, \pi / 6)$} & Grid & $L^{2}$ error & $p$ \\
\hline & $64 \times 64$ & $4.18 \times 10^{-3}$ & \multirow{3}{*}{$\begin{array}{l}3.07 \\
3.63\end{array}$} \\
\hline & $128 \times 128$ & $4.99 \times 10^{-4}$ & \\
\hline & $256 \times 256$ & $4.02 \times 10^{-5}$ & \\
\hline
\end{tabular}

Table 1: $L^{2}$ error and convergence rate $p$ of classical and optimised SBP operators.

adaptive fourth order Runge-Kutta method with a sufficiently small error to not have a significant impact on the experiment.

We begin by studying the convergence rates of the operators through a short-time simulation, where the experiment is run to $t=3$. For such short times, the dispersion error is likely very small, even on the coarsest grid, and we may expect that the higher order $\operatorname{SBP}(8,4)$ operator performs better than the optimised operators. The errors in $L^{2}$ norm is shown in Table 1 together with the convergence rates, $p$. Indeed, the convergence rates are near the expected ones, i.e. $p=5$ for $\operatorname{SBP}(8,4)$ and $p=3$ for the optimised operators.

Our next aim is to study the effect of the numerical dispersion on the solution. To separate the dispersive from the dissipative sources of error we introduce the quantity $E_{\text {disp }}$ defined as

$$
E_{\text {disp }}=\sqrt{\left(x_{\min }^{(a)}-x_{m i n}^{(n)}\right)^{2}+\left(y_{\min }^{(a)}-y_{\min }^{(n)}\right)^{2}},
$$

where $(x, y)_{\min }^{(a, n)}$ are the coordinates of the locations of minimum pressure of the analytic and numerical solutions of (19), which we use as the definition 
of the centers of the vortices. Since (20) only depends on the coordinate of minimum pressure, it is a function of the speed of the vortex, and hence estimates the dispersive qualities of the scheme while ignoring the dissipative ones. A procedure for obtaining $E_{\text {disp }}$ is described in [9]. Here, we perform a long-time simulation by running the experiment to $t=60$, corresponding to three full periods. To account for errors arising from the interfaces as well as from the interior of the domain, we use an average, $\bar{E}_{d i s p}$, over the final (third) period to quantify the dispersion error, that is

$$
\bar{E}_{\text {disp }}=\frac{1}{\left|\mathcal{P}_{3}\right|} \sum_{n \in \mathcal{P}_{3}} E_{\text {disp }}\left(t_{n}\right) .
$$

Here, $\mathcal{P}_{3}$ is the set of discrete time steps constituting the third period of the simulation, while $\left|\mathcal{P}_{3}\right|$ denotes its cardinality. Note that $\mathcal{P}_{3}$ changes depending on the grid and operator used due to the adaptive nature of the Runge-Kutta integrator.

To stabilise the simulation over long time intervals, a small amount of artificial dissipation is added. Here we use an artificial dissipation operator of order eight in the interior and order four near domain interfaces. This operator consists of an undivided difference scheme, approximating the eighth spatial derivative operation. Thus, the impact of these operators scale with the grid spacing; see [35] for details. The influence of this operator is controlled by the weight factor $\epsilon$.

The dispersion errors $\bar{E}_{\text {disp }}$ are listed in Table 2 together with the convergence rates $p$. Here we have used $\epsilon=1$ as a weight factor for the artificial dissipation. Note that the convergence rates are similar to those seen for the $L^{2}$ error in Table 1 , i.e. close to $p=5$ for $\operatorname{SBP}(8,4)$ and close to $p=3$ for the optimised operators.

On the fine grids, where small wavenumbers dominate the solution, using the higher order $\operatorname{SBP}(8,4)$ operator results in the smallest errors. However, clearly the optimised operators all outperform $\operatorname{SBP}(8,4)$ on the coarse $64 \times$ 64 grid, like they are designed to do. This is remarkable since it suggests that error reduction can be obtained using the new operators even when information about the wavenumbers involved is highly restricted. Apparently $\xi_{\max }=\pi / 4$ is a suitable choice of optimisation region for this particular problem. Finding the optimal $\xi_{\max }$ for a given problem may be a delicate task. However, as the experiment demonstrates, improvements compared to the non-optimal stencils can be made even with limited knowledge of the solution. 


\begin{tabular}{|c|c|c|c|}
\hline \multirow{4}{*}{$\operatorname{SBP}(8,4)$} & Grid & $\bar{E}_{\text {disp }}$ & $p$ \\
\hline & $64 \times 64$ & $1.05 \times 10^{-2}$ & \\
\hline & $128 \times 128$ & $6.10 \times 10^{-4}$ & 4.11 \\
\hline & $256 \times 256$ & $1.30 \times 10^{-5}$ & \\
\hline \multirow{4}{*}{$\operatorname{SBP}(4,2,2,8, \pi / 4)$} & Grid & $\bar{E}_{\text {disp }}$ & $p$ \\
\hline & $64 \times 64$ & $5.84 \times 10^{-3}$ & \multirow{3}{*}{$\begin{array}{l}2.68 \\
3.99\end{array}$} \\
\hline & $128 \times 128$ & $9.12 \times 10^{-4}$ & \\
\hline & $256 \times 256$ & $5.75 \times 10^{-5}$ & \\
\hline \multirow{4}{*}{$\operatorname{SBP}(4,2,2,8, \pi / 5)$} & Grid & $\bar{E}_{\text {disp }}$ & $p$ \\
\hline & $64 \times 64$ & $6.44 \times 10^{-3}$ & \multirow{3}{*}{$\begin{array}{l}2.85 \\
3.66\end{array}$} \\
\hline & $128 \times 128$ & $8.92 \times 10^{-4}$ & \\
\hline & $256 \times 256$ & $7.05 \times 10^{-5}$ & \\
\hline \multirow{4}{*}{$\operatorname{SBP}(4,2,2,8, \pi / 6)$} & Grid & $\bar{E}_{\text {disp }}$ & $p$ \\
\hline & $64 \times 64$ & $7.23 \times 10^{-3}$ & \multirow{3}{*}{$\begin{array}{l}3.00 \\
3.44\end{array}$} \\
\hline & $128 \times 128$ & $9.04 \times 10^{-4}$ & \\
\hline & $256 \times 256$ & $8.35 \times 10^{-5}$ & \\
\hline
\end{tabular}

Table 2: Period averaged dispersion error $\bar{E}_{\text {disp }}$ and convergence rate $p$ of classical and optimised SBP operators. 


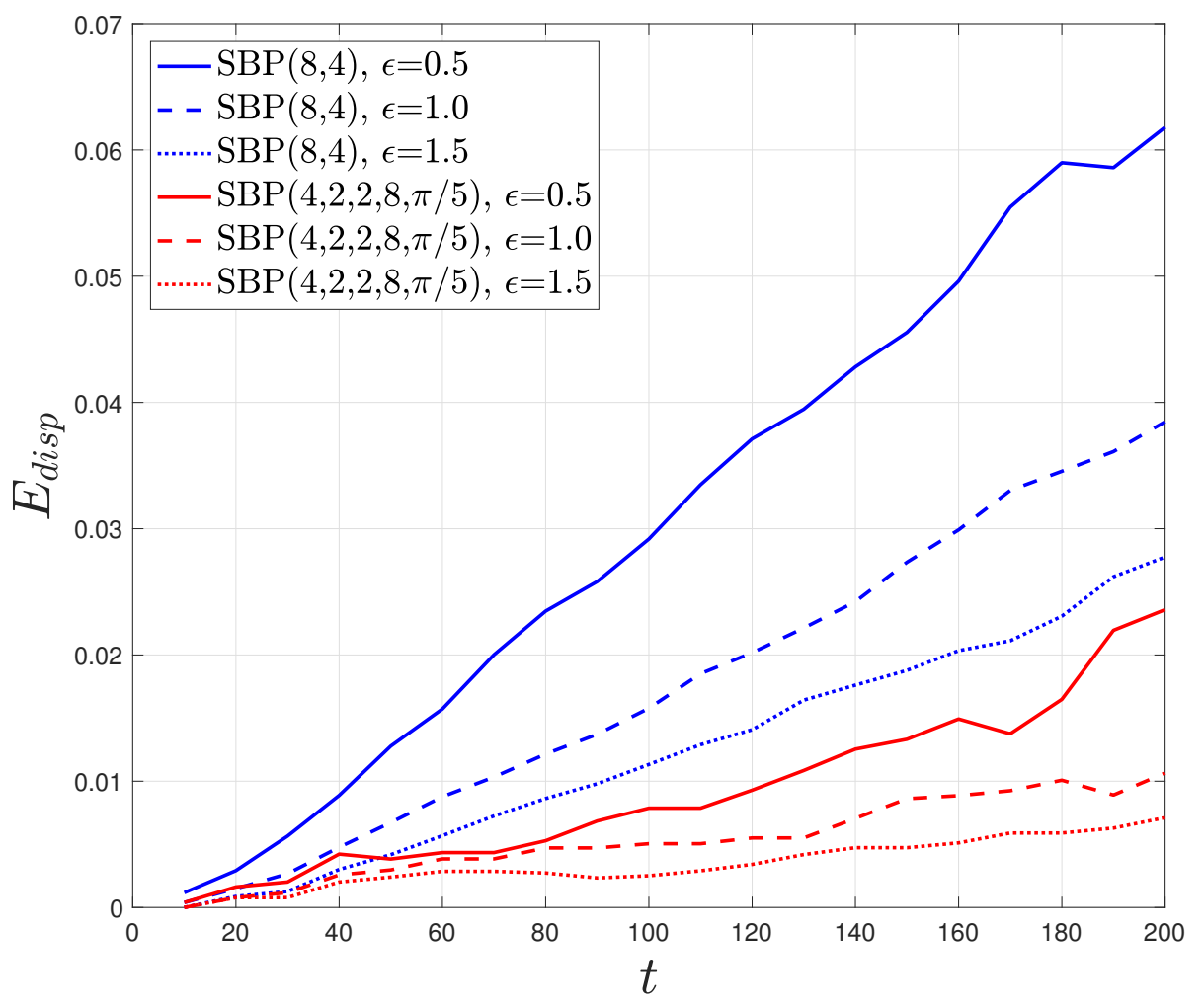

Figure 8: Dispersion error development for a $\operatorname{SBP}(8,4)$ and $\operatorname{SBP}(4,2,2,8, \pi / 5)$ using various amounts of artificial dissipation (AD).

Though $E_{\text {disp }}$ is determined from the speed of the solution, it is in fact not entirely independent of the amount of added artificial dissipation, $\epsilon$. This is due to the fact that the artificial dissipation primarily dissipates modes with large wavenumbers [35]. Since these are the modes causing the dispersion error, a larger $\epsilon$ will in general reduce $E_{\text {disp }}$ (but increase dissipative errors). Fig. 8 illustrates this phenomenon for $\operatorname{SBP}(8,4)$ and $\operatorname{SBP}(4,2,2,8, \pi / 5)$ using $\epsilon \in\{0.5,1.0,1.5\}$. Here we have run the experiment on the $64 \times 64$ grid to time $t=200$, corresponding to ten passes through the numerical interface. It is evident that for long time propagation the advantage of the optimised operators becomes increasingly pronounced, even when a substantial amount of artificial dissipation is added. If we continue to increase $\epsilon$, the dispersion error will reduce further, even for long times. However, this introduces a 
substantial dissipative error and smears the numerical solution. Eventually, the resolution capacity for small scale physical features will be lost. We will investigate this problem further in the next section.

\subsection{The Taylor-Green vortex}

The previous experiment suggests that artificial dissipation should be accounted for in the choice of optimisation region $\xi_{\max }$ since there is little purpose in extending the optimisation to wavenumbers that are heavily damped. In this final example we will see that for an overly damped problem, the choice of operator may have little impact on the outcome since dissipative errors dominate the solution. However, we will also see that the optimised SBP operators may be used with less artificial dissipation than the classical ones, leaving more high wavenumber modes intact, that can be accurately resolved.

In this final example we consider the Taylor-Green vortex (TGV). The TGV flow in a three-dimensional periodic box is a model for the non-linear transfer of kinetic energy among eddies with a large range of sizes. Velocity shear generates kinetic energy, which is dissipated by the viscosity at smaller scales. Thus, the TGV provides a model problem for the evolution of a turbulent flow and the energy transfer to successively smaller spatial scales.

The evolution of the TGV flow has been described in [36]. Initially the behaviour is that of an inviscid flow as the vortices begin to roll-up. Here, artificial dissipation will be required to stabilise the numerical solution. At later times, coherent structures break down and eventually the flow becomes fully turbulent. The solution is primarily dominated by dissipative effects arising from the internal friction forces. As small scale structures evolve, high wavenumber content enters the flow.

The governing equations for the TGV is the three-dimensional compressible Navier-Stokes equations with constant physical properties and a low Mach number, $M_{\infty}=0.1$. We let the fluid be a perfect gas at Reynolds number $R e=1600$ with heat capacity ratio $\gamma=1.4$ and Prandtl number $\operatorname{Pr}=0.71$. The initial temperature field is assumed to be constant. 
The initial conditions are given by

$$
\begin{aligned}
u & =+V_{0} \sin \left(\frac{x}{L}\right) \cos \left(\frac{y}{L}\right) \cos \left(\frac{z}{L}\right) \\
v & =-V_{0} \cos \left(\frac{x}{L}\right) \sin \left(\frac{y}{L}\right) \cos \left(\frac{z}{L}\right) \\
w & =0 \\
p & =1+\frac{V_{0}^{2}}{16}\left(\cos \left(\frac{2 x}{L}\right)+\cos \left(\frac{2 y}{L}\right)\right)\left(\cos \left(\frac{2 y}{L}\right)+2\right),
\end{aligned}
$$

where $u, v, w$ and $p$ respectively represent the flow field in the $x, y$ and $z$ directions, and the pressure. Here $V_{0}=0.1$ and $L=2 \pi$ is the dimension of the periodic box. The duration of the flow is taken to be $20 t_{c}$ where $t_{c}=L / V_{0}$ is the characteristic convective time.

As a measure of the quality of the schemes we compute the kinetic energy of the solution and compare it to data from a direct numerical simulation obtained using a dealiased pseudo-spectral code with vanishingly small numerical dissipation and dispersion errors, run on a $512^{3}$ point grid [37, 38]. Additionally we compute the kinetic energy dissipation rate.

We discretise the problem on a $64 \times 64 \times 64$ grid and enforce periodicity using SAT terms across numerical interfaces. This grid is coarse compared to the smallest structures apparent in the solution. We solve the problem using the fourth order accurate $\operatorname{SBP}(8,4)$ operator, as well as operators of first, second and third order optimised for wavenumbers up to $\xi_{\text {max }}=\pi / 2$; $\operatorname{SBP}(2,1,3,8, \pi / 2), \operatorname{SBP}(4,2,2,8, \pi / 2)$ and $\operatorname{SBP}(6,3,1,8, \pi / 2)$. All simulations use a Courant number of 0.1 . The same artificial dissipation operator as we used in the Euler vortex problem is applied here. As before, the strength of the dissipation is governed by the parameter $\epsilon$.

Initially we set $\epsilon=0.5$. The results are shown in Fig. 9. Clearly all solutions are overly dissipative, which is particularly obvious during the transition to turbulence from $t / t_{c} \approx 5$ to $t / t_{c} \approx 10$. After this, small scale structures with high wavenumbers are surpressed by the artificial dissipation to which the operators respond differently. The fast dissipation rate continues for the lower order operators that resolve the small scales well, whereas the higher order operators with poorer resolution capacity appear to leave more energy in the computational domain.

We now repeat the experiment using a minimal amount of artificial dissipation. The appropriate value for $\epsilon$ is found to be 0.2 for the classical $\operatorname{SBP}(8,4)$ while, remarkably, the optimised operators can all be used with 


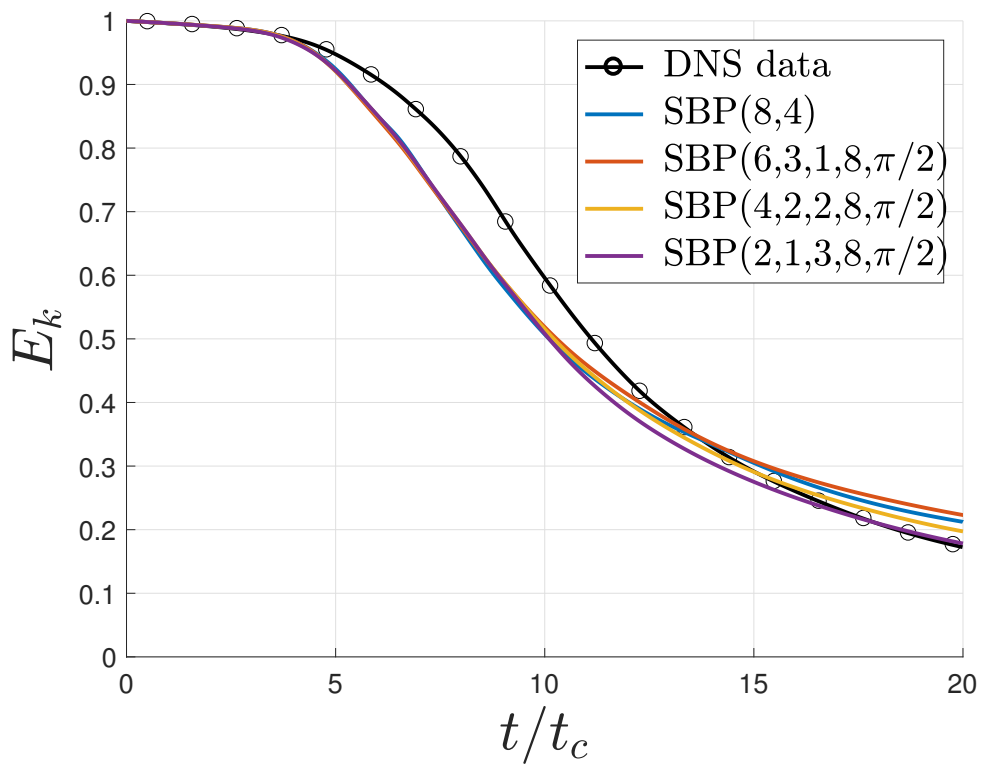

(a) Kinetic energy evolution.

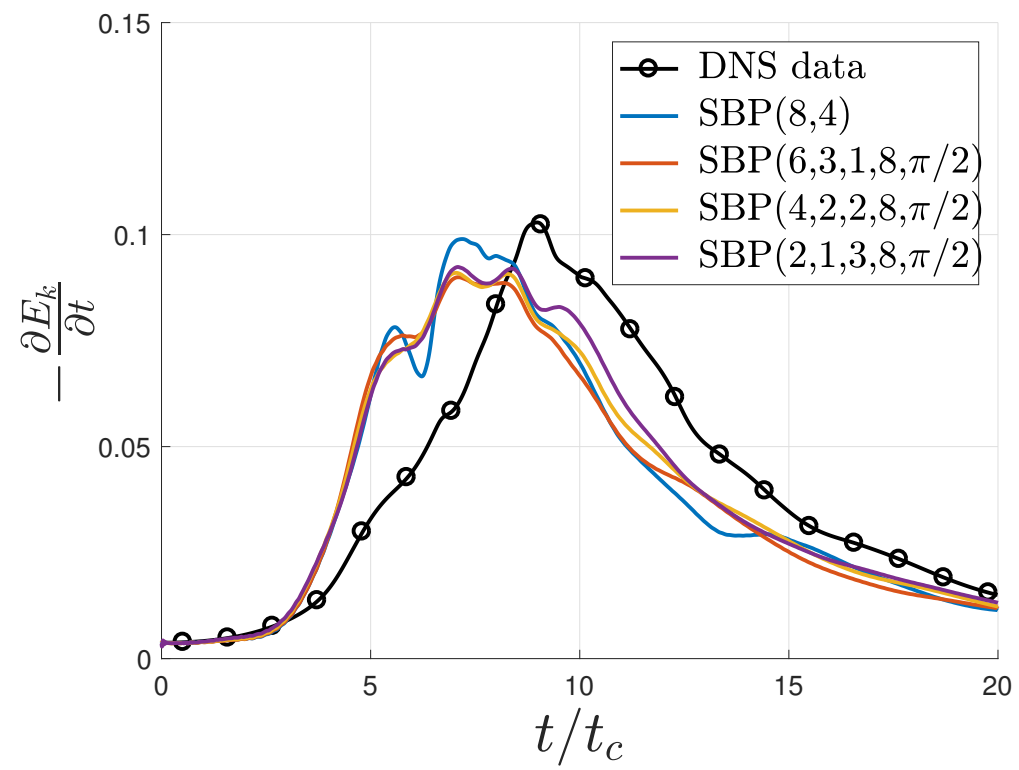

(b) Kinetic energy dissipation rate.

Figure 9: Kinetic energy evolution and dissipation rate using $\epsilon=0.5$. 


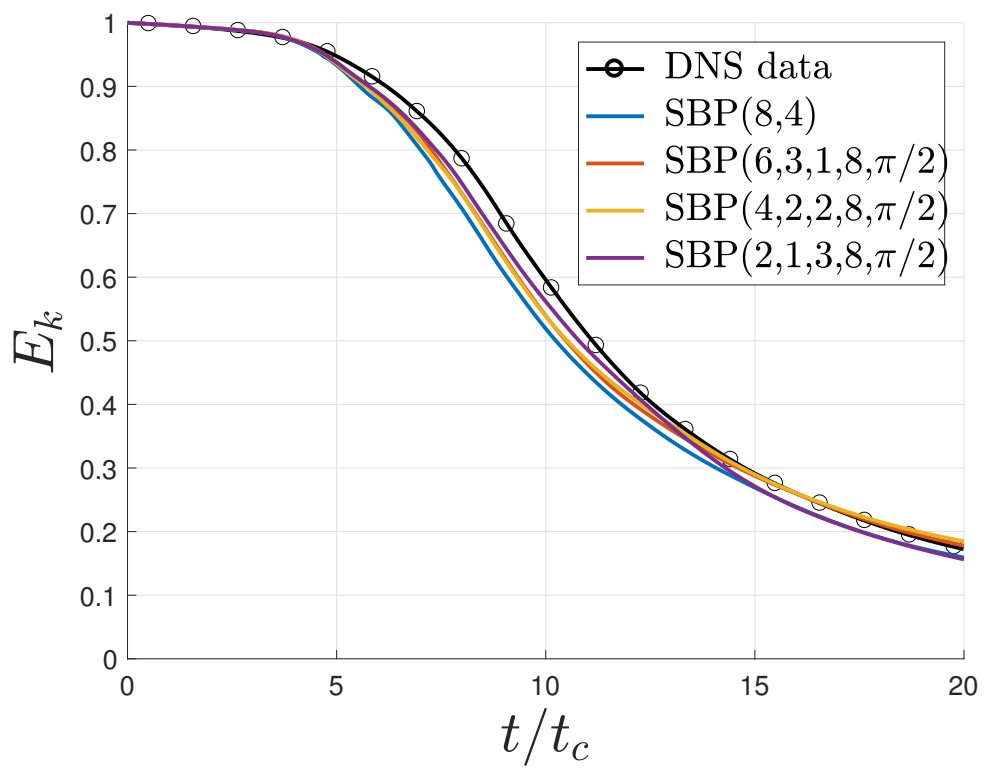

(a) Kinetic energy evolution.

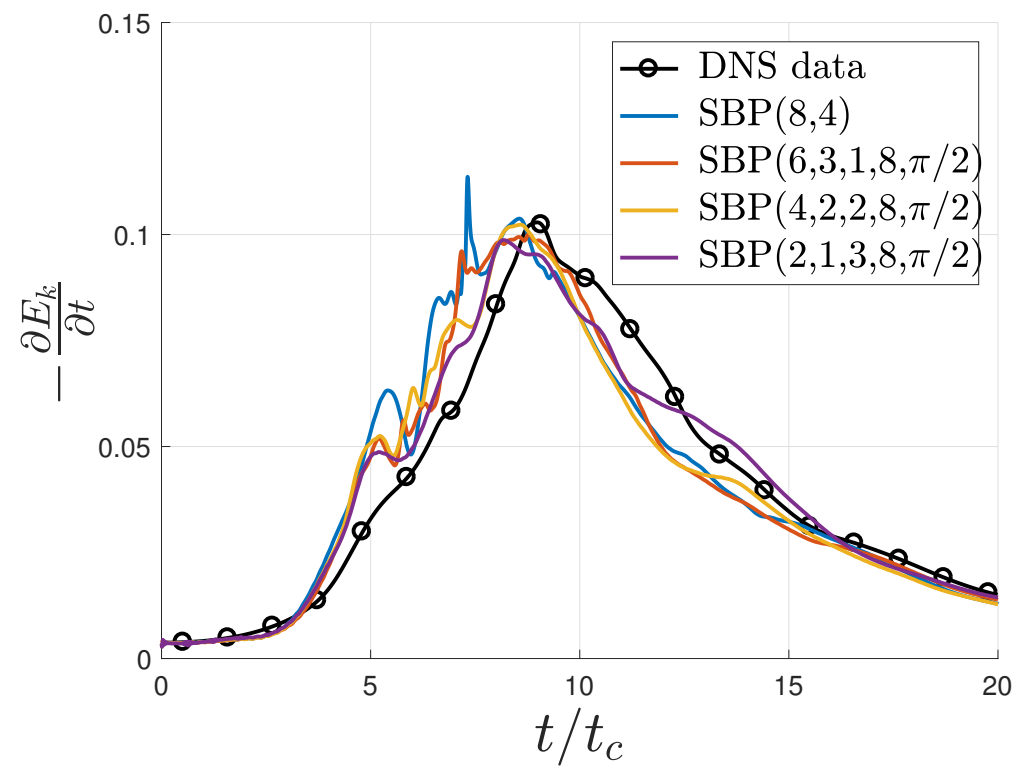

(b) Kinetic energy dissipation rate.

Figure 10: Kinetic energy evolution and dissipation rate using minimal artificial dissipation. 
$\epsilon=0.05$. The results are shown in Fig. 10. The classical $\operatorname{SBP}(8,4)$ operator displays an erratic behaviour of the kinetic energy dissipation rate in the transition to turbulence. On the other hand, the optimised operators handle this rather well. In particular, $\operatorname{SBP}(4,2,2,8, \pi / 2)$ and $\operatorname{SBP}(6,3,1,8, \pi / 2)$ capture the peak dissipation at $t / t_{c} \approx 9$ within a close margin. Note also that the latter two operators preserve the kinetic energy with high precision in the later stages of the simulation; from $t / t_{c} \approx 13$ and onwards. Here, large wavenumbers become progressively more influential, though still with a dominance of the smaller wavenumbers [39]. It thus appears that $\operatorname{SBP}(4,2,2,8, \pi / 2)$ and $\operatorname{SBP}(6,3,1,8, \pi / 2)$ provide the right balance between high order (approximating modes with smaller wavenumbers) and high resolution to capture most of the solution even at a very coarse grid.

\section{Conclusions}

An inaccurate representation of the analytic dispersion relation by finite difference stencils may, for problems involving high wavenumbers or large time scales, be the dominant source of error. To remedy this, a variety of central difference schemes have been presented in the literature that preserves the dispersion relation of the governing equations. In this paper we have developed Summation-by-Parts operators that extend this dispersion relation preservation to stencils near interfaces, thus allowing for a much broader range of problems to be solved with high resolution while retaining stability and high order.

We have optimised the SBP operators by enforcing that Fourier modes with a range of wavenumbers should be accurately approximated on a given grid parametrised by the step size $\Delta x$. This has been shown to be equivalent to finding the stencil coefficients that minimise the largest dispersion error associated with the stencil near numerical interfaces. The problem of constructing such operators is quasiconvex and can therefore be solved using any standard convex optimisation package in a matter of seconds.

Comparisons of optimised operators with a higher order classical SBP operator of the same computational complexity has revealed that significant error reductions can be obtained for solutions with high wavenumber content, or propagation problems over large spatiotemporal intervals. This is expressed partly in terms of a severe underestimation of the wavespeed of high frequency waves, and partly in terms of reflections at numerical interfaces that subsequently become trapped in the computational domain. Both 
these effects are significantly reduced when the dispersion error is properly minimised.

Similar improvements are seen for non-linear systems of equations as has been demonstrated by solving the Euler vortex problem. It has been shown that operators optimised for several different wavenumber ranges can reduce the dispersion error on coarse grids for a given problem. This suggests that the optimisation procedure might be useful even if knowledge of the (normalised) numerical wavenumbers involved is limited. For long time propagation, the benefits of the optimised operators become increasingly pronounced, even in the presence of significant artificial dissipation.

Finally, by solving the Taylor-Green vortex it has been demonstrated that the optimised operators can be used with less artificial dissipation than classical ones. Consequently, we can allow, and accurately preserve, numerical solutions containing Fourier modes with larger wavenumbers, hence improving accuracy. This suggests that optimised SBP operators could be used as tools for turbulence simulations when only coarse grids are available.

The operators introduced in this paper are designed for problems where computational resources are limited, but some knowledge of the spectral content of the solutions is available. They are not intended to replace higher order operators, which indeed are superior when fine grids are available. Nonetheless, the experiments suggests that on moderately or very coarse grids, optimised SBP operators provide an improvement even when knowledge of the spectral content is limited. For a given problem, finding the sweet spot among order of accuracy, stencil width (or number of free parameters) and optimisation regime may be a delicate task and we have not attempted to do so here. In fact, for some problems it may be advisable to change operator mid-simulation. It is an interesting topic, still requiring further research, to obtain in-simulation estimates of dominating wavenumbers without ruining computational efficiency. 


\section{References}

[1] D. W. Zingg, H. Lomax, H. Jurgens, An optimized finite-difference scheme for wave propagation problems, AIAA paper 93(0459) (1993).

[2] J. Lighthill, The final panel discussion, in: J. Hardin, M. Hussaini (Eds.), Computational Aeroacoustics, Springer-Verlag, New York, 1993, pp. 499-513.

[3] M. S. Fox-Rabinovitz, Computational dispersion properties of 3d staggered grids for a nonhydrostatic anelastic system, Monthly Weather Review 124 (1995) 498-510.

[4] A. Taflove, Computational Electrodynamics: The Finite-Difference Time-Domain Method, Artech House, Boston, 1995.

[5] A. Sei, A family of numerical schemes for the computation of elastic waves, SIAM J. Sci. Comput. 16 (1995) 898-916.

[6] D. W. Zingg, Comparison of high-accuracy finite-difference methods for linear wave propagation, SIAM J. Sci. Comput. 22 (2000) 476-502.

[7] C. Bogey, C. Bailly, A family of low dispersive and low dissipative explicit schemes for flow and noise computations, Journal of Computational Physics 194 (2004) 194-214.

[8] G. Cunha, S. Redonnet, A novel optimization technique for explicit finite-difference schemes with application to aeroacoustics, Int. J. Numer. Meth. Fluids 78 (2015) 189-216.

[9] V. Linders, J. Nordström, Uniformly best wavenumber approximations by spatial central difference operators, Journal of Computational Physics 300 (2015) 695-709.

[10] H.-O. Kreiss, G. Scherer, Finite element and finite difference methods for hyperbolic partial differential equations, Aspects of Finite Elements in Partial Differential Equations, Academic Press, Inc. (1974).

[11] H.-O. Kreiss, G. Scherer, On the existence of energy estimates for difference approximations of hyperbolic systems, Technical report, Dept. of Scientific Computing, Uppsala University (1977). 
[12] B. Strand, Summation by parts for finite difference approximations for d/dx, Journal of Computational Physics 110 (1994) 47-67.

[13] M. Svärd, J. Nordström, Review of summation-by-parts schemes for initial-boundary-value problems, Journal of Computational Physics 268 (2014) 17-38.

[14] M. H. Carpenter, J. Nordström, D. Gottlieb, A stable and conservative interface treatment of arbitrary spatial accuracy, Journal of Computational Physics 148 (1999) 341-365.

[15] C. Tam, J. Webb, Dispersion-Relation-Preserving finite difference schemes for computational acoustics, Journal of Computational Physics 107 (1993) 262-281.

[16] S. Johansson, High order difference approximations for the linearized Euler equations, Lic. Uppsala Universitet, Department of Information Technology (2004).

[17] B. Müller, S. Johansson, Strictly stable high order difference approximations for computational aeroacoustics, Comptes Rendus Mecanique 333 (2005) 699-705.

[18] B. Müller, High order numerical simulation of aeolian tones, Computers \& Fluids 37 (2008) 450-462.

[19] M. H. Carpenter, D. Gottlieb, S. Abarbanel, Time-stable boundary conditions for finite-difference schemes solving hyperbolic systems: Methodology and application to high-order compact schemes, Journal of Computational Physics 111(2) (1994).

[20] D. C. Del Rey Fernández, J. E. Hicken, D. W. Zingg, Review of summation-by-parts operators with simultaneous approximation terms for the numerical solution of partial differential equations, Computers \& Fluids 95 (2014) 171-196.

[21] M. Carpenter, D. Gottlieb, Spectral methods on arbitrary grids, Journal of Computational Physics 129 (1996) 74-86.

[22] G. J. Gassner, A skew-symmetric discontinuous galerkin specral element discretization and its relation to SBP-SAT finite difference methods, SIAM J. Sci. Comput. 35 (2013) 1233-1253. 
[23] D. C. Del Rey Fernandez, P. D. Boom, D. Zingg, A generalized framework for nodal first derivative summation-by-parts operators, Journal of Computational Physics 266 (2014) 214-239.

[24] S. Eriksson, Q. Abbas, J. Nordström, A stable and conservative method for locally adapting the design order of finite difference schemes, Journal of Computational Physics 230 (2011) 4216-4231.

[25] M. Svärd, On coordinate transformations for summation-by-parts operators, J. Sci. Comput. 20 (2004) 29-42.

[26] J. Nordström, Conservative finite difference formuations, variable coefficients, energy estimates and artificial dissipation, J. Sci. Comput. 29 (2006) 375-404.

[27] M. Svärd, J. Nordström, On the order of accuracy for difference approximations of initial-boundary value problems, Journal of Computational Physics 218 (2006) 333-352.

[28] S. Boyd, L. Vandenberghe, Convex Optimization, Cambridge University Press, Cambridge, United Kingdom, 7th edition, 2009.

[29] D. Eppstein, Quasiconvex programming, Combinatorial and Computational Geometry, MSRI Publications 52 (2005).

[30] M. Grant, S. Boyd, Cvx: Matlab software for disciplined convex programming, version 2.0 beta, http://cvxr.com/cvx, 2013.

[31] M. Grant, S. Boyd, Graph implementations for nonsmooth convex programs, recent advances in learning and control (a tribute to $\mathrm{m}$. vidyasagar), http://stanford.edu/ boyd/graph_dcp.html, 2008.

[32] K. Mattsson, M. Svärd, M. Carpenter, J. Nordström, High-order accurate computations for unsteady aerodynamics, Computers \& Fluids 36 (2007) 636-649.

[33] K. Mattsson, M. Almquist, M. Carpenter, Optimal diagonal-norm SBP operators, Journal of Computational Physics 264 (2014) 91-111.

[34] G. Erlebacher, M. Hussaini, C. Shu, Interaction of a shock with a longitudinal vortex, J. Fluid Mech. 337 (1997) 129-153. 
[35] K. Mattsson, M. Svärd, J. Nordström, Stable and accurate artificial dissipation, Journal of Scientific Computing 21 (2004) 57-79.

[36] M. E. Brachet, D. I. Meiron, S. A. Orszag, B. Nickel, R. H. Morf, U. Frisch, Small-scale structure of the taylor-green vortex, Journal of Fluid Mechanics 130 (1983) 411-452.

[37] W. M. van Rees, A. Leonard, D. I. Pullin, P. Koumoutsakos, A comparison of vortex and pseudo-spectral methods for the simulation of periodic vortical flows at high reynolds numbers, Journal of Computational Physics 230 (2011) 2794-2805.

[38] K. Hillewaert, 3rd international workshop on high order methods, https://www.grc.nasa.gov/hiocfd/, 2015.

[39] J. DeBonis, Solutions of the taylor-green vortex problem using highresolution explicit finite difference methods, AIAA paper 382 (2013) 2013. 